\title{
Tutoría entre pares. Experiencia del programa asesoría pedagógica estudiantil. Universidad Católica de Santiago de Guayaquil*
}

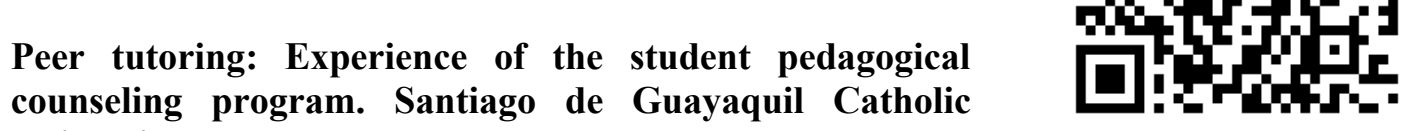
University

Yadira Blakman Briones**

Elba Bermúdez Reyes ${ }^{* * *}$

\section{Resumen}

El aprendizaje centrado en el estudiante, paradigma de las últimas reformas universitarias, recomienda crear ambientes de aprendizaje en los cuales, los estudiantes sean protagonistas de su propio proceso de aprendizaje, a través de altos niveles de participación y autonomía. Dentro del proceso de innovación educativa, la Universidad Católica de Santiago de Guayaquil (UCSG), implemento la tutoría entre pares, que es una metodología pedagógica, desarrollada por estudiantes de alto desempeño, que acompañan, dan soporte y ayudan a sus compañeros, en un área disciplinar nudo crítico para que puedan mejorar su rendimiento académico. Los objetivos de este trabajo de investigación fueron, determinar el nivel de efectividad del programa apoyo pedagógico estudiantil (APE) en la modalidad tutoría entre pares, evaluar el

\footnotetext{
* Artículo original derivado del Proyecto "Competencias docentes universitarios" Financiado por la Universidad Católica Santiago de Guayaquil entre agosto 2018 y mayo 2019

** Docente Universitaria. Universidad Católica Santiago de Guayaquil. Guayaquil, Ecuador. E-mail: yadira.blakman@cu.ucsg.edu.ec. ORCID: http://orcid.org/0000-0002-2491-5796.

*** Docente Universitaria. Universidad Católica Santiago de Guayaquil. Guayaquil, Ecuador. E-mail: elba.bermudez@cu.ucsg.edu.ec. ORCID: http://orcid.org/0000-0003-3261-3218
}

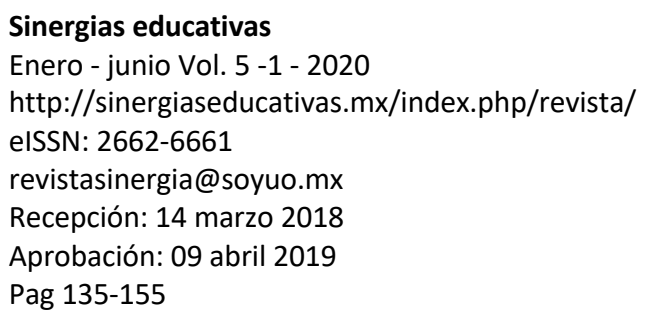

Disponible en

http://www.redalyc.org/articulo.oa?id=57356143200 11

Atribución/Reconocimiento-NoComercialCompartirlgual 4.0 Licencia Pública Internacional CC BY-NC-SA 4.0 
desempeño académico de los estudiantes de la muestra bajo estudio y rediseñar el programa APE luego de diez años de su implementación, para fortalecer el rendimiento académico de los estudiantes de los tres primeros ciclos. La metodología consistió en realizar un diagnóstico de la eficacia del programa APE, se trató de un estudio cuantitativo, descriptivo y transversal, en el que participaron 312 estudiantes tutorados por el programa y 58 docentes de las asignaturas que representan nudos críticos, en las 9 facultades de la UCSG. No se ha investigado sobre naturaleza multicausal que inciden en el bajo desempeño académico. Como resultado se conoce que el programa APE en la UCSG, alcanzo un nivel de efectividad satisfactorio. Se concluye que la tutoría entre pares es una estrategia que contribuye al mejoramiento del rendimiento académico.

Palabras clave: acompañamiento académico, Tutoría entre pares, Asesoría Pedagógica, Estudiante Tutor.

\section{Abstract}

Student-centered learning, a paradigm of the latest university reforms, recommends creating learning environments in which students are protagonists of their own learning process, through high levels of participation and autonomy. This approach of student centrality has been collected by the pedagogical educational model of the Catholic University of Santiago de Guayaquil (UCSG), without neglecting the important role that the teacher plays as a tutor, facilitator and mediator in the learning process. Within the process of educational innovation, the UCSG implemented peer tutoring, which is a pedagogical methodology, developed by highperformance students, who accompany, support and help their peers, in a critical knot discipline area, so that they can improve your academic performance The objectives of this research work were to determine the level of effectiveness of the student pedagogical support program (APE) in the peer tutoring modality, evaluate the academic performance of the students in the sample under study and redesign the APE program after ten years of its implementation, to strengthen the academic performance of the students of the first three cycles. The methodology consisted of making a diagnosis of the effectiveness of the APE program, it was a quantitative, descriptive and cross-sectional study, in which 312 students tutored by the 
program participated and 58 teachers of the subjects representing critical nodes, in the 9 faculties of the UCSG. $t$ has not been investigated about multicausal nature that affects the low academic performance. As a result, it is known that the Student Pedagogical Advisory Program (APE) at UCSG, reached a satisfactory level of effectiveness. It is concluded that peer tutoring is a strategy that contributes to the improvement of academic performance.

Key words: academic Accompaniment, Peer Tutoring, Pedagogical Advice, Student Tutor.

\section{Introducción}

Las últimas reformas universitarias propuestas por la UNESCO, se orientan hacia el desarrollo de la máxima implicación de los estudiantes en su propio aprendizaje y la adquisición de competencias, que no solo les permitan formarse sino poder aprender a lo largo de la vida, en esa misma línea de pensamiento Lobato \& Nagore (p. 379, 2016) agregaron "como sujeto activo que aprende autónomamente competencias personales y profesionales". Este planteamiento requiere de la creación de ambientes de aprendizajes participativos, autónomos, centrado en el estudiante, de manera que él se convierta en el protagonista de su propio proceso de aprendizaje.

El ingreso a la universidad para un estudiante, implica un cambio en su proceso de desarrollo psicosocial; la vida universitaria en muchos casos significa dejar la zona de confort para asumir nuevas responsabilidades, entre ellas, la autonomía sobre su reciente etapa y todo el proceso de desarrollo que esto conlleva, algunos deben separarse de sus familias o residir en una nueva ciudad, establecer nuevas relaciones interpersonales o tomar decisiones importantes acerca de su proyecto de vida profesional y personal, si este paso no se realiza en forma adecuada, podrían derivar en crisis, que afectarán sus actividades sociales y académicas, provocando en situaciones más graves el abandono de los estudios.

El bajo rendimiento académico, la deserción y el rezago, es otra de las dificultades que afrontan las universidades del país, situación que también se evidencia en los últimos años en la UCSG, especialmente 
con los estudiantes de los primeros años, afectando la eficiencia terminal de las carreras. En este contexto, la problemática no solo preocupa a las autoridades educativas del sistema de educación superior, sino también a las autoridades gubernamentales que han tenido que generar políticas públicas para elevar la calidad de la educación y lograr los resultados esperados.

Para Bianculli y Marchal (p. 381, 2013) esta situación se ha convertido en un "obstáculo y un problema estructural, que está dando origen a la emergencia de políticas educativas vinculadas a favorecer el ingreso, la retención y la graduación de los alumnos". Por lo tanto, institucionalizar un programa de tutorías entre pares, de acuerdo con la experiencia de otras universidades y la nuestra, favorecerá el desarrollo integral del estudiante, al ser una estrategia metodológica educativa mejorará el rendimiento académico y la calidad de la educación.

En la tutoría entre pares, el docente es el guía para el estudiante-tutor, a quién necesita afianzar y fortalecer en su papel; requiere que el profesor lo apoye, lo respalde, lo oriente, y le permita obtener el conocimiento fundamental y las herramientas tutoriales necesarias que tendrá que desplegar a lo largo de esta labor pedagógica. Servirá para disminuir el rezago y el abandono educativo en las aulas universitarias; favorecerá su desarrollo psicosocial y mejorará la calidad de la educación superior, de ahí se deriva la importancia de conocer los factores que influyen en el desempeño académico para adecuar nuestro programa de apoyo pedagógico estudiantil APE, de acuerdo a las necesidades de los estudiantes y las exigencias académicas.

Como precedente, se han encontrado varios estudios sobre asesoría pedagógica y tutoría universitaria, generalmente encausados al acompañamiento del docente hacia el estudiante. En el caso particular de Ecuador, si bien es cierto es un tema de interés, hay muy pocas experiencias investigativas sobre la tutoría entre pares en los espacios universitarios, sin embargo en los últimos años, el tema se ha convertido en un asunto de interés para las instituciones de educación superior. 
Algunos estudios han indicado que las actividades colaborativas entre compañeros mejoran el rendimiento académico, por otra parte, se ha señalado que la tutoría entre pares aporta al desarrollo académico integral, pues se potencian las habilidades cognitivas, sociales y afectivas, necesarias para el desempeño académico, el aprendizaje autónomo, la autoestima y el desarrollo de la comunicación como competencia social básica. Se ha evidenciado que el trabajo colaborativo a través de la ayuda ofrecida entre compañeros, desarrolla las capacidades para construir un conocimiento valioso al elaborar sus propios significados, de la misma forma proporciona confianza y autonomía en la autorregulación de los procesos.

Otros autores mencionan que favorece la integración de los estudiantes a la vida universitaria la utilidad de proporcionarles información y orientación sobre en asuntos académicos, administrativos y socio-afectivos. La literatura estudiada, da cuenta que la tutoría universitaria de pares, se inscribe en el contexto de la innovación educativa y los procesos de reforma de la educación superior en la que nos encontramos inmersos.

\section{El constructivismo en la educación universitaria y la acción tutorial}

El constructivismo educativo propone un paradigma donde el proceso de enseñanza se percibe y se lleva a cabo como proceso dinámico, participativo e interactivo del sujeto, de modo que el conocimiento sea una auténtica construcción manejada por la persona que aprende. Cuando un sistema educativo adopta el constructivismo como línea psicopedagógica se orienta a llevar a cabo la enseñanza orientada a la acción, lo que implica un cambio en todos los niveles, plantea que el proceso enseñanza aprendizaje se dé en un ambiente creativo y de innovación, mientras más innovador es el docente tutor, más despertará la conciencia creativa en sus estudiantes. Con esta orientación se favorece el desarrollo de los procesos cognitivos y creativos, para que posteriormente el estudiante despliegue con autonomía e independencia en su práctica profesional. Así, el aprender a aprender, es clave para la acción tutorial y retoma especial importancia al abordar el desarrollo de habilidades; en ese plano los aprendizajes se conciben como la 
vinculación que hace el estudiante de los conocimientos con las experiencias previas.

\section{El desempeño académico}

El desempeño académico es una dimensión de las capacidades que tiene el estudiante, indica lo que éste ha aprendido a lo largo de todo el proceso formativo, además evidencia la capacidad del mismo para responder a los estímulos educativos. En este sentido, el rendimiento académico está vinculado a la habilidad para adquirir conocimientos, por lo tanto, es imprescindible elevar el desempeño académico de los estudiantes, ya que el hecho de no poder alcanzar el promedio requerido, genera inconvenientes como el rezago educativo, esto implica que le tomará más tiempo culminar su carrera, en ocasiones estas deficiencias son tales que el estudiante se ve obligado a desertar del sistema educativo, tal como lo señala Hernández y Pozo (1999. p.45), "puede originar en él insatisfacción personal, desmotivación e incluso baja autoestima ante la imposibilidad de concluir con éxito sus estudios".

\section{La tutoría universitaria}

La tutoría universitaria, según Álvarez, (2014) es una función de acompañamiento, de orientación y de apoyo al estudiante, en su proceso de personalización de los aprendizajes y del desarrollo de las competencias, tanto a nivel personal como profesional; adopta diferentes modelos dependiendo de los contextos, la cultura universitaria, de las políticas y normas educativas, recursos disponibles y necesidades de los estudiantes.

Durán (2009) citando a Cannon-Bowers y Salas, menciona que en este contexto el desarrollo de las competencias individuales, toma protagonismo la competencia cooperativa, entendida como un conjunto de conocimientos, habilidades y actitudes necesarias para un trabajo en equipo efectivo. De esta manera, la cooperación se constituye como una competencia fundamental en la sociedad del conocimiento que facilita, no solo el desarrollo de habilidades y actitudes autónomas; sino que constituye el motor de aprendizaje que utiliza pedagógicamente las diferencias entre los estudiantes como un elemento a favor del aprendizaje. 
La formación centrada en el estudiante y la adopción de una mayor calidad en la educación superior, conducen al enorme esfuerzo de implicación de los estudiantes, al buen conocimiento de sus potencialidades para que a partir de ahí poder gestionar gradual y apropiadamente su propio aprendizaje y el desarrollo de competencias que les permitan formarse y poder aprender a lo largo de la vida.

\section{Tutoría entre pares en la universidad}

Para asegurar el desarrollo exitoso de la competencia cooperativa, Duran (2009) citando a O'Neil, Chung y Brown mencionan que es necesario tener en cuenta dos procesos que suceden de manera similar: uno orientado a la tarea propiamente dicha y otro encausado de manera específica al trabajo en equipo. Dichos procesos, principalmente el que se refiere al desarrollo del trabajo en equipo, requiere de una manera $u$ otra la participación activa y comprometida de los estudiantes.

Aprender a trabajar con otras personas, sintiéndose parte de un equipo, es un hecho que implica la práctica de algunas habilidades relacionadas con la cooperación y que necesitan de la participación e interacción entre estudiantes para poder desarrollar la competencia.

Según los autores Boud, Cohen y Sampson (2001) citados por Duran (2009), las habilidades a desplegar al interior del grupo son: el desarrollo de la escucha activa, el razonamiento y la argumentación adecuada de las ideas propias, pensar de forma crítica y reflexiva, formular preguntas críticas, proponer distintas soluciones a un problema para poder construir conocimiento con otros; organizar el propio aprendizaje y el de los compañeros, tomar decisiones personales y de equipo, resolver los conflictos de manera negociada y asertiva; autoevaluarse y coevaluarse unos a otros a lo largo del proceso, aprender a ofrecer apoyo y saber pedir ayuda, así como valorar el propio trabajo y el de los compañeros. Todas estas competencias requieren la participación mínima de dos estudiantes para ponerlas en práctica y desarrollarlas en el contexto educativo para posteriormente poderlas transferir a contextos profesionales, donde el desempeño del trabajo en equipo es considerado esencial. 
El aprendizaje entre pares o iguales, es definido por Boud et al., (2001,p 7); como "situaciones educativas en que los estudiantes tienen oportunidades recíprocas de aprender y enseñar; de aprender de y con los otros tanto en situaciones formales como informales (puede ser mutuamente beneficioso y aúna la capacidad de compartir el conocimiento, las ideas y las experiencias entre los participantes, promoviendo el aprendizaje interdependiente o mutuo" así mismo mencionaron que también es una valiosa oportunidad de aprendizaje para el profesorado, ya que le anima a cambiar sus concepciones de enseñanza y aprendizaje a partir del cambio de rol en el aula de clase, dejando su rol estelar, a guía o mediador de los procesos educativos.

En contextos instructivos formales dicen:

Se entiende la tutoría entre iguales como un método de aprendizaje entre iguales basado en la creación de parejas, con una relación asimétrica (derivada del rol de tutor o de tutorado que desempeñan respectivamente), con un objetivo común, compartido y conocido (adquisición de una competencia académica), que se logra a través de un marco de relación planificado por el profesor.

La tutoría de pares, según Seoane, et al, (2014, p. 3):

Posee un gran potencial socio afectivo y el hecho de que en la tutoría intervengan estudiantes, beneficia a ambos actores participantes del proceso, otorgando una serie de ventajas, entre las que se destacan: se crea un contexto de aprendizaje y comunicación favorable, un clima positivo; se favorece la autoestima y estimula el desarrollo de competencias de liderazgo, capacidad instructiva y trabajo colaborativo.

En este mismo sentido Vygotsky (1962), menciona que "los tutorados se benefician del aprendizaje, ya que reciben una ayuda constante, personalizada y ajustada a su zona de desarrollo próximo".

En este contexto de proximidad se genera un clima de confianza que permite a los tutorados poder plantear dudas y equivocarse sin temor, cosa que resulta más difícil cuando la interacción se produce 
directamente con el profesor, reduciéndose el estrés y la ansiedad (Melero y Fernández, 1995).

Los tutorados, asumiendo el rol que les ha sido asignado, cumplen con las atribuciones de buenos estudiantes e intentan demostrar su aprendizaje mostrando una mayor motivación y compromiso. Se trata pues de ofrecer oportunidades a todos los estudiantes, tutores y tutorados, para que aprendan enseñándose unos a otros aprovechando positivamente sus diferencias (Duran, 2009).

En el ámbito de la educación superior, mencionaron que los beneficios de las tutorías de pares son diversos, entre ellos, se distingue su aporte pedagógico y social, así como el mejoramiento de la calidad de la experiencia universitaria, al crearse vínculos y fortalecer relaciones entre compañeros, ayudando al desarrollo social y cognitivo.

\section{Materiales y métodos}

Se trata de un estudio descriptivo, transversal, de enfoque cuantitativo, con análisis cualitativo de los resultados obtenidos en la investigación, algunos de ellos fueron recogidos en cuadros estadísticos; esta articulación de los datos, ofrecieron mejores posibilidades de estudio, análisis y reflexión. Con respecto a los métodos y las técnicas de recolección de datos, se realizaron encuestas a los estudiantes y docentes; se implementó un muestreo no probabilístico intencional, puesto que los participantes eran estudiantes de primero a tercer ciclo, atendidos por el programa APE en las nueve facultades de la Universidad Católica de Santiago de Guayaquil, durante el período 2015 - 2016 y docentes de las asignaturas que constituyeron el nudo crítico de tipo académico. La población ascendió a un total de 312 estudiantes y 58 docentes.

La primera fase del proyecto consistió en medir la influencia del programa y tuvo como objetivo determinar el nivel de efectividad de la Asesoría Pedagógica Estudiantil (APE), en las diferentes Facultades de la UCSG, realizando una evaluación de los logros académicos de los estudiantes asesorados. 
La muestra bajo estudio fue del $58 \%$ del universo de estudiantes atendidos por APE, se seleccionó intencionalmente a los estudiantes, en primera, segunda y tercera matrícula, con calificaciones inferiores a 6,5 puntos, que fueron derivados y atendidos por el programa por ser considerados en riesgo académico. El $42 \%$ de estudiantes que también fueron atendidos por el programa, no fueron seleccionados porque tienen buen rendimiento y asisten a los talleres para mantener sus promedios de calificaciones y becas. Se creó una base de datos, con la información de los estudiantes en riesgo académico, derivados $\mathrm{y}$ atendidos por APE, matriculados en las nueve facultades que oferta UCSG, en la que se determinaron: las asignaturas nudo críticos, las notas o calificaciones obtenidas durante los semestres A y B - 2015 y A y $\mathrm{B}$-2016, número de matrícula, número de estudiantes atendidos y número de estudiantes que mejoraron sus notas $\mathrm{y}$ aprobaron las materias. Estos datos fueron ordenados y registrados por facultades, carreras y semestres. Con los datos estudiados se procedió a su análisis cuantitativo y con ello se pudo obtener el nivel de efectividad de la ejecución del programa. De toda la información ordenada y racionalizada en la base de datos, se obtuvo un cuadro estadístico, que refleja el porcentaje de efectividad logrado por el programa durante el período mencionado; para esto fue necesario la elaboración y aplicación de una escala tipo Likert y se determinaron tres baremos: Muy Satisfactorio, Satisfactorio y Poco Satisfactorio. Se determinó una escala numérica de medición del 10\% al 100\% como indicador del grado de intensidad de la eficiencia del programa, en el primer baremo Muy Satisfactorio, se consideraron los máximos puntajes de la escala, comprendidos entre $90 \%$ y $100 \%$, en el segundo baremo Satisfactorio, se consideraron los puntajes medios comprendidos entre el $50 \%$ y el $80 \%$ y finalmente, en el tercer baremo Poco Satisfactorio, a los puntajes inferiores de la escala, esto es del 10\% al $40 \%$.

La segunda fase del proyecto fue analizar el desempeño académico, el objetivo de esta fase consistió en evaluar a los estudiantes y docentes de la muestra bajo estudio, a través de encuestas, para elaborar un cuadro comparativo que permita contrastar sus percepciones. 
El enfoque metodológico utilizado en esta fase de la investigación, fue abordado de forma cuantitativa, las encuestas fueron aplicadas con la colaboración de las asesoras pedagógicas de cada una de las nueve Facultades de la UCSG, para el análisis de los datos se utilizaron medidas de tendencia central de la estadística descriptiva, como la mediana y la media. La información recolectada, fue apoyada en dos cuestionarios con preguntas cerradas y de elección múltiple, donde los encuestados escogieron la respuesta según su criterio o apreciación. Los datos recogidos fueron organizados en una tabla de distribución de frecuencias, la misma que permitió el análisis e interpretación de las características que describen y que no son evidentes en el conjunto de datos no procesados, de acuerdo a los valores de la variable.

La tercera fase de la investigación consistió en mejorar el modelo de acompañamiento pedagógico actual, en base a los resultados obtenidos en las fases anteriores, al análisis y estudio de investigaciones y experiencias previas de universidades iberoamericanas con la finalidad de mejorar el desempeño de los estudiantes de los tres primeros ciclos.

\section{Resultados}

Los resultados parciales de la primera fase de la investigación permitieron evidenciar que el programa APE, en una escala tipo Likert, ha alcanzado un nivel de eficiencia Satisfactorio. Sin embargo, queda pendiente, en lo que resta de la presente investigación, determinar qué hacer para que el logro de eficiencia pase a Muy Satisfactorio, que es el nivel de aspiración que persigue la UCSG, para el bienestar de sus estudiantes. 


\section{Tabla 1}

Fase I: Cuadro estadístico para evaluar el nivel de efectividad del programa APE a través de los logros académicos de los estudiantes asesorados

\begin{tabular}{|c|c|c|c|}
\hline FACULTAD & CARRERA & \begin{tabular}{|l|} 
Promedio de \\
efectividad \\
año 2015 \\
\end{tabular} & $\begin{array}{l}\text { Promedio de } \\
\text { efectividad } \\
\text { año } 2016 \\
\end{array}$ \\
\hline \multirow{3}{*}{$\begin{array}{lr}\text { Facultad } & \text { de } \\
\text { Arquitectura } & \text { y } \\
\text { Diseño } & \end{array}$} & Arquitectura & 43,50 & 56,00 \\
\hline & Diseño de Interiores & 54,50 & 45,00 \\
\hline & $\begin{array}{l}\text { Gestión Gráfica y } \\
\text { Publicitaria }\end{array}$ & 60,00 & 59,50 \\
\hline \multirow{3}{*}{$\begin{array}{l}\text { Facultad de Artes } \\
\text { y Humanidades }\end{array}$} & Música & 81,00 & 56,50 \\
\hline & $\begin{array}{l}\text { Producción y Artes } \\
\text { audiovisuales }\end{array}$ & 49,50 & 69,50 \\
\hline & Lengua Inglesa & 62,50 & 50,00 \\
\hline \multirow{4}{*}{$\begin{array}{l}\text { Facultad de } \\
\text { Ciencias } \\
\text { Económicas y } \\
\text { Administrativas }\end{array}$} & Economía & 60,50 & 50,00 \\
\hline & \begin{tabular}{|l|} 
Gestión Empresarial \\
Internacional
\end{tabular} & 48,50 & 58,00 \\
\hline & Contabilidad & 45,00 & 63,00 \\
\hline & $\begin{array}{l}\text { Administración de } \\
\text { Empresas }\end{array}$ & 56,00 & 56,50 \\
\hline $\begin{array}{l}\text { Facultad de } \\
\text { Ciencias Médicas }\end{array}$ & Medicina & 63,39 & 73,22 \\
\hline \multirow{5}{*}{$\begin{array}{l}\text { Facultad de } \\
\text { Especialidades } \\
\text { Empresariales }\end{array}$} & Turismo & 43,00 & 63,50 \\
\hline & Marketing & 86,00 & 67,50 \\
\hline & $\begin{array}{|ll|}\text { Comercio } & \mathrm{y} \\
\text { Finanzas } & \\
\text { Internacionales } & \\
\end{array}$ & 55,00 & 50,50 \\
\hline & Emprendimiento & 78,50 & 54,50 \\
\hline & Ventas & 100,00 & 100,00 \\
\hline \multirow{3}{*}{$\begin{array}{l}\text { Facultad de } \\
\text { Filosofía, Letras } \\
\text { y Ciencias de la } \\
\text { Educación }\end{array}$} & Pedagogía & 81,82 & 76,92 \\
\hline & Psicología & 75,90 & 81,93 \\
\hline & $\begin{array}{l}\text { Comunicación } \\
\text { Social }\end{array}$ & 71,82 & 62,22 \\
\hline \multirow{3}{*}{$\begin{array}{l}\text { Facultad de } \\
\text { Ingeniería }\end{array}$} & Ingeniería Civil & 45,50 & 31,00 \\
\hline & \begin{tabular}{|l|} 
Ingeniería en \\
Sistemas \\
Computacionales \\
\end{tabular} & 47,50 & 39,50 \\
\hline & Derecho & 80,96 & 71,79 \\
\hline
\end{tabular}




\begin{tabular}{|c|c|c|c|}
\hline $\begin{array}{l}\text { Facultad de } \\
\text { Jurisprudencia y } \\
\text { Ciencias Sociales } \\
\text { y Políticas }\end{array}$ & $\begin{array}{l}\text { Trabajo Social y } \\
\text { Desarrollo Humano }\end{array}$ & 76,45 & 75,64 \\
\hline \multirow{5}{*}{$\begin{array}{l}\text { Facultad Técnica } \\
\text { para el Desarrollo }\end{array}$} & $\begin{array}{l}\text { Ingeniería } \\
\text { Agropecuaria }\end{array}$ & 41,38 & 54,95 \\
\hline & $\begin{array}{l}\text { Medicina } \\
\text { Veterinaria } \\
\end{array}$ & 62,22 & 56,25 \\
\hline & $\begin{array}{l}\text { Ingeniería } \\
\text { Agroindustrial }\end{array}$ & 30,00 & 33,33 \\
\hline & $\begin{array}{l}\text { Ingeniería en Electro } \\
\text { Mecánica }\end{array}$ & 40,48 & 30,21 \\
\hline & $\begin{array}{l}\text { Ingeniería en } \\
\text { Telecomunicaciones }\end{array}$ & 71,67 & 77,52 \\
\hline
\end{tabular}

Elaboración propia.

\section{Tabla 2}

Fase II: Evaluar desde la perspectiva de docentes y estudiantes, factores que frecuentemente dificultan el desempeño académico de los estudiantes.

CUADRO COMPARATIVO DE ESTUDIANTES Y DOCENTES \#1

\begin{tabular}{|c|c|c|}
\hline & DOCENTES & ESTUDIANTES \\
\hline & $\begin{array}{l}\text { ¿Cuáles son los factores más } \\
\text { frecuentes de dificultan el } \\
\text { aprendizaje } \\
\text { estudiantes? }\end{array}$ & $\begin{array}{l}\text { ¿Cuáles son los factores más } \\
\text { frecuentes por los que el } \\
\text { aprendizaje de las asignaturas } \\
\text { en las que recibió } \\
\text { acompañamiento pedagógico } \\
\text { se les dificultaba? }\end{array}$ \\
\hline Categorías & $\mathrm{F}_{\mathrm{r} \%}$ & $\mathrm{~F}_{\mathrm{r} \%}$ \\
\hline Poco interés en el estudio & 82,76 & 17,31 \\
\hline $\begin{array}{l}\text { Insuficiencia en la } \\
\text { comprensión lectora }\end{array}$ & 37,93 & 19,23 \\
\hline $\begin{array}{l}\text { Carencia de técnicas de } \\
\text { estudio }\end{array}$ & 51,72 & 39,10 \\
\hline Vacíos de años anteriores & 75,86 & 46,15 \\
\hline $\begin{array}{l}\text { Metodología empleada por } \\
\text { el profesor }\end{array}$ & 6,90 & 51,60 \\
\hline $\begin{array}{ll}\text { Recursos } & \text { didácticos } \\
\text { inapropiados } & \end{array}$ & 3,45 & 16,67 \\
\hline
\end{tabular}




\begin{tabular}{l|l|l} 
Insuficiente uso de las tics & 12,07 & 9,62 \\
\hline $\begin{array}{l}\text { Insuficientes recursos } \\
\text { didácticos del docente }\end{array}$ & 6,90 & 27,88 \\
\hline Otros & 12,07 & 6,09
\end{tabular}

Elaboración propia.

En el análisis de este cuadro, es importante resaltar los porcentajes más altos, en las dos categorías Docentes y Estudiantes, para así poder obtener una visión más amplia de la situación. Con respecto al motivo por el cual se dificulta el aprendizaje, el $82,76 \%$ de los docentes encuestados manifiestan que se debe al poco interés de los estudiantes en el estudio. Mientras que, la dificultad más frecuente de acuerdo a la percepción de1 51,60\% de los estudiantes, en aquellas asignaturas que recibió acompañamiento pedagógico, radica en la metodología empleada por el docente.

Este punto de vista opuesto, debe llamar a la reflexión a los actores del proceso educativo, porque este al ser sistémico, se convierte en una situación estrechamente vinculante. La función docente es un elemento fundamental del aprendizaje, como importante es su rol de mediador entre un plan de estudios y lo que acontece en las aulas. Así mismo la centralidad del estudiante reviste importancia ya que él es corresponsable de su proceso de aprendizaje. De manera que hay que optar por incorporar al ambiente de aprendizaje, metodologías de acción participativas en clase.

Habría que recordar que una práctica educativa implica una actividad necesariamente interpersonal, en la que cada uno de los actores que participan tiene una perspectiva muy particular del otro en función de su comportamiento, y de la forma en que conciben cada una de las situaciones escolares que viven de manera cotidiana en el contexto educativo.

Continuando con el análisis, se puede apreciar que el 75,86\% de los docentes mencionaron que otro de los factores que frecuentemente dificulta el aprendizaje en los estudiantes, se debe al vacío de conocimientos de años anteriores. Coincidiendo con la percepción de los docentes, la mayoría de los estudiantes, el 46,15\% 
manifestaron que se debe también a los vacíos de conocimientos que arrastran de años anteriores.

Este hallazgo valida la teoría de Ausubel 1983 quien sostenía que existen dos principios de aprendizaje que operan en el aula de clase: el de repetición - aprendizaje significativo y el principio de recepción por descubrimiento, estos principios son válidos siempre que el estudiante relacione el nuevo conocimiento que le facilite el docente con su estructura cognitiva y que el material suministrado sea de interés para él.

Cuando el docente presenta una nueva información a un estudiante que no tiene conocimientos previos, el aprendizaje que se produce es un aprendizaje repetitivo, memorístico que se caracteriza porque no existe ninguna relación entre las experiencias previas del estudiante y ésta, el alumno se ve en la necesidad de memorizarlo porque no alcanza a comprender el significado, la coherencia, la validez y su correspondiente organización interna.

El análisis de los resultados de la encuesta, permite evidenciar que los estudiantes la hora de ejecutar sus actividades académicas, la mayoría $47,12 \%$ observan videos y el $42,31 \%$ tiende a memorizar lo transmitido por el docente; se puede inferir que el estudiante de los primeros ciclos, memoriza contenidos, pero utiliza más las herramientas tecnológicas en el proceso de aprendizaje, lo que no es novedad porque son nativos digitales. Mientras que $60,34 \%$ de los docentes implementan.

talleres, otro 55,17\%, utiliza el trabajo colaborativo como metodología en clase, sin- embargo solo un $34,48 \%$ de docentes utiliza herramientas tecnológicas audio visuales en clase.

Los hallazgos encontrados en esta investigación han sido un insumo importante para la UCSG porque permitió conocer la opinión de los estudiantes y docentes sobre los factores que inciden en el rendimiento académico, se conoce que son varios, pero esencialmente hay que prestarle atención a la metodología utilizada por el profesor y a los vacíos de conocimiento de años anteriores en los estudiantes de los primeros ciclos, sin dejar de lado a la 
motivación y el uso de las Tics, que contribuyen y facilitan la experiencia tutorial.

Ha posibilitado al docente conocer las necesidades de sus estudiantes, en este caso se requiere de su disposición a implementar nuevas estrategias didácticas apoyadas por las TIC, las que prometen abrir canales de comunicación más eficientes, más participativos y de forma permanente.

Se ha demostrado que el interés del estudiante por aprender, puede hacer que este tenga un alto o bajo desempeño académico, pero también es muy importante la forma de enseñar. La capacidad creativa e innovadora del docente también es un factor decisivo para transmitir el conocimiento, se debe valer de herramientas tecnológicas que contribuyan a mejorar el proceso de aprendizaje y motive el interés por el estudio.

Fase III:

Consistió en proponer el rediseño del modelo de acompañamiento pedagógico estudiantil existente, para mejorar el desempeño de los estudiantes de los tres primeros ciclos, en base a los resultados obtenidos en las fases anteriores y al estudio de investigaciones y experiencias previas de universidades iberoamericanas.

Este rediseño se sustenta en el marco jurídico de la Ley Orgánica de Educación Superior, las Asesorías Pedagógicas Estudiantiles, se constituyen en la estrategia para apoyar el proceso formativo integral de los estudiantes en todas las instituciones de Educación Superior Como producto de la presente investigación, se propone incorporar las siguientes acciones de mejoras al programa actual:

- Funcionar con lineamientos oficializados en la Universidad.

- Estar considerado en el Plan Estratégico Institucional.

- Estar orientado con una visión, misión y objetivos estratégicos regulados institucionalmente.

- Plantear una estructura organizativa institucionalizada. 
- Crear perfiles y descripciones de funciones reconocidos institucionalmente.

- Proceso de selección de Ayudantes Pedagógicos en base a: alto rendimiento académico, competencia comunicativa, actitud proactiva para el trabajo colaborativo, liderazgo y emocionalmente estable para el trabajo autónomo de los estudiantes.

- Incorporar al modelo tutoría entre pares, el apoyo y orientación del docente para que sea el mentor del estudiante-tutor.

- Llevar a la asesoría pedagógica estudiantil a plataformas virtuales.

- Desarrollar instrumentos para evaluar los estilos de aprendizaje de estudiantes atendidos por el programa.

- Capacitar en temas específicos al equipo pedagógico de acuerdo a los requerimientos del nuevo modelo de APE.

- Evaluar periódicamente la efectividad del programa (estrategia, metodología y valoración del desempeño de los miembros de APE).

\section{Discusión}

De acuerdo a investigaciones previas y bibliografías revisadas en base de datos en español, los programas acompañamiento pedagógico estudiantil, modalidad tutoría de pares, dan cuenta que, todas ejercen sus actividades desde diferentes ámbitos: docencia, académico, gestión administrativa y social; están alineadas a un marco jurídico, producto de las últimas reformas educativas y poseen políticas que la orientan y normas que la rigen. Investigar en este campo ha permitido que el conocimiento avance, hacia la construcción de las bases teóricas y metodológicas del acompañamiento pedagógico estudiantil y sus modelos: tutoría docente y tutoría de pares en espacios universitarios. La experiencia de la UCSG ha confirmado la teoría que el acompañamiento pedagógico contribuye notablemente al mejoramiento del rendimiento académico de los estudiantes que participan de estos espacios. El programa tributa a la formación integral de los estudiantes, en sus diferentes enfoques, unos al proceso de 
adaptación e integración del estudiante al sistema de estudio universitario y otros contribuyen a la reducción de la deserción estudiantil y mejoran la tasa de eficiencia terminal de las universidades.

Concordamos con otros autores que la facilidad de comunicación y de interacción entre pares, ha sido el elemento esencial que genera confianza y el ambiente de aprendizaje ideal para mejorar el desempeño académico, al ofrecerles un entorno seguro $\mathrm{y}$ participativo a los estudiantes. Se coincide con experiencias previas, acerca de la necesidad de institucionalizar la acción tutorial, la cual debe contar con el respaldo de los directivos de la institución, estar alineada a una visión, a una política, a objetivos, a regulaciones, tener una estructura y poseer una planificación estratégica.

\section{Conclusiones}

Los hallazgos encontrados en esta investigación han sido un insumo importante para la UCSG porque permitió conocer la opinión de los estudiantes y docentes sobre los factores que inciden en el rendimiento académico, se conoce que son varios, pero esencialmente hay que prestarle atención a la metodología utilizada por el profesor y a los vacíos de conocimiento de años anteriores en los estudiantes de los primeros ciclos, sin dejar de lado a la motivación y el uso de las Tics, que contribuyen y facilitan la experiencia tutorial.

El estudio ha permitido conocer, qué piensa, qué cree, cómo concibe el estudiante su relación con los docentes y cómo estas concepciones afectan la forma en que él se acerca al conocimiento. Ha posibilitado al docente conocer las necesidades de sus estudiantes, en este caso se requiere de su disposición a implementar nuevas estrategias didácticas apoyadas por las TIC, las que prometen abrir canales de comunicación más eficientes, más participativos $\mathrm{y}$ de forma permanente.

Lo que no se ha investigado es acerca de la naturaleza multicausal de los factores que inciden en el desempeño académico de un estudiante, cómo estos diversos factores personales, familiares y socioeconómicos pueden incidir en un bajo rendimiento académico, 
no se han analizado variables psicológicas como el autoestima, la ansiedad y crisis depresivas, pueden incidir en el fracaso educativo, que fueron causas para que algunos estudiantes sean derivados al programa de apoyo pedagógico estudiantil.

Una vez concluida la investigación, podemos establecer que el programa APE, de acuerdo a las respuestas obtenidas en las encuestas realizadas a los usuarios en las 9 facultades de la UCSG, logró un Nivel Satisfactorio.

De acuerdo al segundo objetivo planteado, se determina que entre docentes y estudiantes hay un punto de vista en el cual ambos coinciden, los vacíos de conocimientos de años anteriores afectan al proceso de aprendizaje y al desempeño académico.

El tercer objetivo de acuerdo a los resultados de los datos obtenidos en la investigación se concluye que no solo es necesario un rediseño del programa, también es necesario institucionalizarlo para que alcance un mayor nivel de eficacia.

\section{Referencias}

Álvarez, P. (2014). La función tutorial del profesorado universitario: Una nueva

competencia de la labor docente en el contexto del EEES. Revista Portuguesa de Pedagogía, 47(2), 85-106.

Albanaes, P.; Marques de Sousa, F. y Patta Bardagi, M. (2015). Programas de tutoría y mentoría en universidades brasileñas: Un estudio bibliométrico. Revista de Psicología, 33(1), 21-56.

Ausubel, D.P. (1976).Psicología educativa: un punto de vista cognoscitivo. México,

Editorial Trillas. p. 58.

Bianculi, K. y Marchal, M. (2013). Las tutorías universitarias: estudio de caso:

Programa de Tutorías PACENI de la Facultad de Ciencias Económicas y Sociales de la UNMdP. Mar del Plata: Universidad Nacional de Mar del Plata. 
Boud, D., Cohen, R. y Sampson, J. (Eds.) (2001). Peer learning in higher education. Londres: Kogan Page.

Durán, D. (2009a). Aprender a cooperar. Del grupo al equipo. En J.I. Pozo y M.P.

Pérez (Eds.), La Psicología del aprendizaje universitario: la formación en competencias. Madrid: Ediciones Morata.

Durán, D. Flores Coll M. (2014). Prácticas de tutoría entre iguales en universidades

del Estado español y de Iberoamérica. Revista Iberoamericana sobre Calidad, Eficacia y Cambio en Educación, REICE. Universitat Autónoma de Barcelona. ISSN: 1696-4713 www.rinace.net/reice/.

Hernández, J. \& Pozo, C. (1999). El fracaso académico en la Universidad: Diseño de un sistema de evaluación y detección temprana. Obtenido de Universidad de La Rioja: https://dialnet.unirioja.es/servlet/articulo? codigo $=2826416$.

Ley Orgánica de Educación Superior LOES (2012). Recuperado de:

http://www.ces.gob.ec/index.php?option=com_phocadownload\&vi ew $=$ category\&id=11:ley-organica-de-educacion-

superior\&Itemid $=137$

Lobato Fraile, C. Nagore Guerra, B. (2016). La tutoría en la educación superior

en Iberoamérica: Avances y desafíos. EDUCAR, vol. 52, núm. 2, 2016, pp. 379-398 Universitat Autònoma de Barcelona. Barcelona, España. Disponible en: http://www.redalyc.org/articulo.oa?id=342146063009

Melero, M.A. y Fernández, P. (1995). La interacción social en contextos educativos. Madrid: Siglo XXI.

Seoane, M. Hernández, O. Novelli, D. Fernandez, M. Piccardo, V. Collazo M. (2017).

Tutoría entre pares: Primera experiencia de curso curricular opcional de la UDELAR. Línea Temática: Prácticas para la reducción del abandono: acceso, integración y planificación Universidad Tecnológica de Panamá. (2014, p. 3).

Vygotsky, L.S. (1962). Thought and language. Cambridge, MA: M.I.T. Press. 
UNESCO (1998) Modernización. Un desafío para la educación. Recuperado de http://unesdoc.unesco.org/images/0008/000884/088427SB.pdf 